\title{
The Esthetic Salutation and Functional Acceptance of Maxillary Superimposed Prosthesis
}

\author{
${ }^{1}$ Vini Rajeev, ${ }^{2}$ Rajeev Arunachalam, ${ }^{3}$ Sivadas Ganapathy, ${ }^{4}$ Vaishanavi Vedam
}

\begin{abstract}
In cases where patients are left with few teeth, clinicians face a dilemma. Psychological satisfaction of the patient by retaining the natural teeth not only provides retention but also helps in the preservation of the alveolar bone and proprioception. Overdenture therapy has a long-term advantage where the forces of mastication are directed along the long axis of the abutment teeth, which provides guidance and support and further prevents the dislodgement of denture during movements. Overdenture design remains one of the best treatment options for patients who do not have the financial ability to support an extensive restorative treatment. This management is a preventive prosthodontic therapy, which can delay or eliminate future prosthodontic problems. This case report is about the prosthetic rehabilitation of a partially edentulous patient using a hybrid overdenture with a double crown for the maxilla and conventional overdenture for the mandible, giving the patient a denture with better esthetics, more support, and retention.
\end{abstract}

Keywords: Double crown, Overdenture, Preventive prosthodontics, Telescopic.

How to cite this article: Rajeev V, Arunachalam R, Ganapathy S, Vedam V. The Esthetic Salutation and Functional Acceptance of Maxillary Superimposed Prosthesis. World J Dent 2016;7(2): 107-110.

\section{Source of support: Nil}

\section{Conflict of interest: None}

\section{INTRODUCTION}

Rehabilitation of partially edentulous jaw can be at risk following the failure of tooth replacement as the resorption of alveolar bone is progressive and permanent. Since bone loss is uncontainable, its maintenance should be gauged. Preventive prosthodontics is the branch of dentistry that is based on the concept of conservation of few remaining natural teeth, thereby preventing bone

\footnotetext{
1-4 Lecturer

${ }^{1}$ Department of Prosthodontics, AIMST University, Bedong Kedah, Malaysia

${ }^{2,3}$ Department of Periodontics, AIMST University, Bedong Kedah, Malaysia

${ }^{4}$ Department of Oral Pathology, AIMST University, Bedong Kedah, Malaysia
}

Corresponding Author: Vini Rajeev, Lecturer, Department of Prosthodontics, AIMST University, Bedong, Kedah, Malaysia Phone: +60143928025, e-mail: vinirajeev09@gmail.com loss. ${ }^{1}$ Overdenture therapy is essentially a preventive prosthodontic notion based on two principles that safeguard the best of oral health: Primarily sustained maintenance of periodontal support around remaining dentition $^{2}$ and secondarily preservation of periodontal sensory mechanisms that gives the patient a sense of perception to touch and pressure, which is not possible using conventional complete dentures. ${ }^{3}$

Overdentures have received an overwhelming response among patients as they give a sense of natural feeling and the adaptation time is less compared to conventional complete dentures. Variants in techniques and theories put forward for using natural teeth to support and stabilize the overdenture design are described in the literature; ${ }^{4}$ however, when it comes to the management of the upper esthetic zone with teeth in bad shape, such as in the case of a fracture, dental caries, or when periodontally compromised, clinicians are exposed to an uphill task of satisfying the patients' demand in terms of esthetics and function. One such novel method that can be employed is the usage of double crowns, which can be fitted into each other, known as maxillary superimposed prosthesis/telescopic dentures. It comprises coping/male telescopic portion luted to the prepared tooth with a secondary crown/female telescopic portion, which is a part of the denture framework and is connected by means of interfacial surface tension over the primary casting. Telescopic anchors provide additional support to the remaining framework in addition to removal of unwanted occlusal forces.

Complete dentures form the ultimate option for rehabilitation in most situations because other options are unsuitable. However, the final treatment result can be improved by the usage of remaining natural dentition in strategic positions for stability and retention of overdentures. Maxillary superimposed prosthesis can also be used as indirect retainers to prevent dislodgement of the distal extension base (DEB) away from the edentulous ridge. ${ }^{5}$ Such a prosthesis transfer forces along the long axis of abutment teeth and gives direction, support, and guard from movements that happen in the oral cavity during operation. ${ }^{6}$ The conservative approach of telescopic overdentures supported by natural teeth can be considered as one of the best treatment options for the edentulous situations, despite recent development in dental implantology. 


\section{CASE REPORT}

A 55-year-old female patient reported to the Department of Prosthodontics, with the complaint of inability to chew and sagging appearance of her face due to missing teeth. The patient was a vocalist by profession and was much concerned about the esthetics and retention of the prosthesis. Extraoral examination suggested a loss of vertical dimension of the face and unsupported lips. The patient had a convex profile, and temporomandibular joint evaluation did not suggest any abnormality. On clinical examination, it was seen that the maxillary ridge had 13,12,11,21, and 22 and the mandibular ridge had 33, 43, and 44. Clinically all the remaining natural teeth were seen to be firm. Periodontal findings were significant with mild amount of calculus and stain present along with Millers class I gingival recession with no pocket formation. ${ }^{7}$ The patient had labially inclined premaxilla and an accompanying severe labial undercut (Fig. 1). The maxillary residual ridge was favorable with adequate bone height and width, and favorable palatal form, while the mandibular ridge was moderately resorbed.

After clinical and radiographic evaluation, a treatment plan in consultation with the patient was devised to retain the remaining teeth and fabricate a telescopic doublecrown overdenture with ridge grip on the maxillary arch opposing tooth supported with a conventional overdenture with short coping on the mandibular arch. The treatment plan included endodontic and prosthodontic phase. The remaining natural teeth were endodontically treated. Once the teeth were asymptomatic, diagnostic impressions were recorded with the help of alginate impression material (Zelgan) for the partially edentulous maxillary and mandibular arches. A tentative jaw relation was done to assess the interarch space, which was found adequate for the proposed treatment plan.

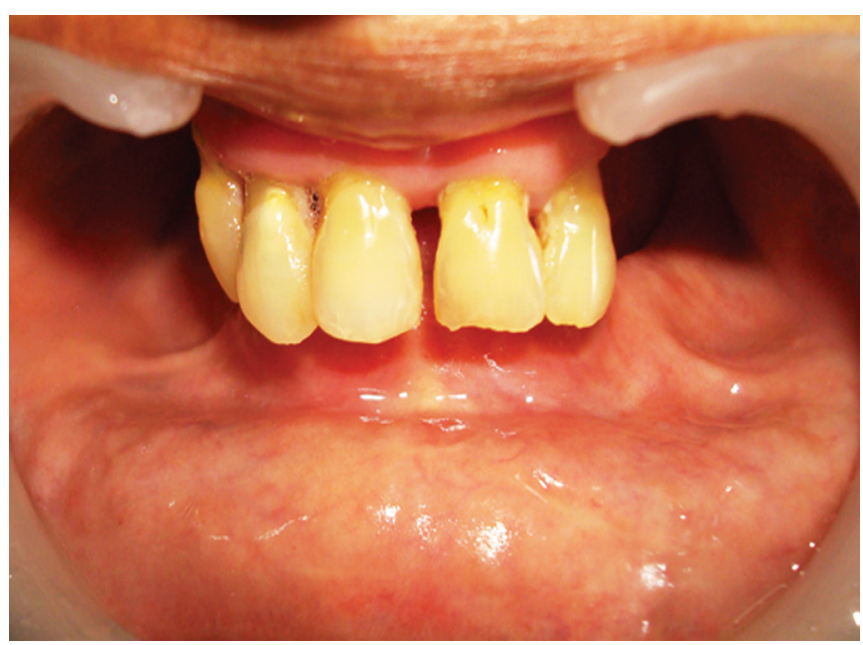

Fig. 1: Preoperative intraoral view
Tooth preparation was done with a chamfer finish line on maxillary anteriors with a taper of approximately 8 to $10^{\circ}$ and overdenture preparation for short coping in lower arch (Fig. 2). After the mouth preparation, gingival retraction was done and an impression was made with addition silicone using the putty-wash technique. The first master model was prepared from the impression for fabrication of the primary copings. In the laboratory, the wax patterns were prepared for the primary copings. The patterns were milled to obtain a frictional surface for retention and cobalt chrome alloy was used for the casting of copings. Once the primary copings were evaluated for fit, they were luted with zinc oxide eugenol on the maxillary teeth and an over-impression was made using the medium viscosity addition silicone impression material and the second master model was fabricated. The second master model together with the primary copings was duplicated and the refractory model was prepared. This model was used for the fabrication of the cast superstructure on the maxillary arch along with secondary coping. In the laboratory, the copings on the second master model were milled. The framework was waxed up, which was then cast using cobalt-chromium alloy along with the secondary coping. After evaluating the fit of the framework in the mouth, it was used for cementing the primary copings in place. The primary copings of both arch were luted with glass ionomer luting cement (Type I; GC Fuji) (Fig. 3). Ceramic layering was done over the secondary copings on the maxillary arch with shade A2. An alginate impression of the lower arch was made for the fabrication of working cast after the cementation of the short copings. Custom tray was fabricated using autopolymerizing resin. A master impression of mandibular arch was made after peripheral tracing using putty consistency silicone and the secondary impression was made using medium viscosity addition silicone. A wax rim was prepared on

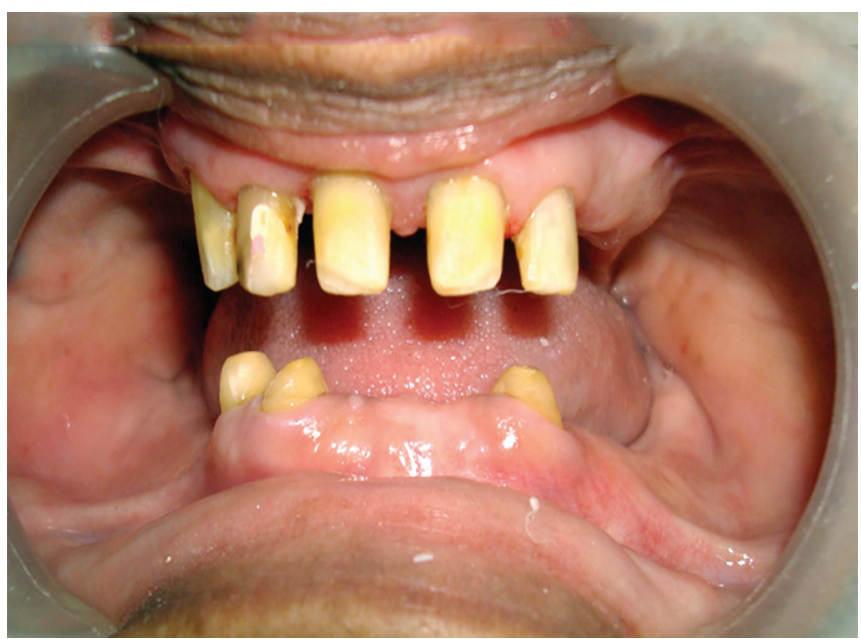

Fig. 2: Mouth preparation 


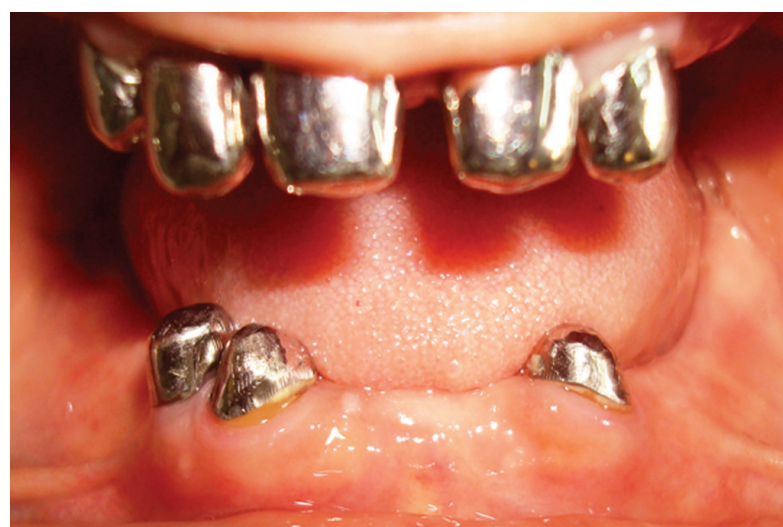

Fig. 3: Intraoral view of cemented primary copings

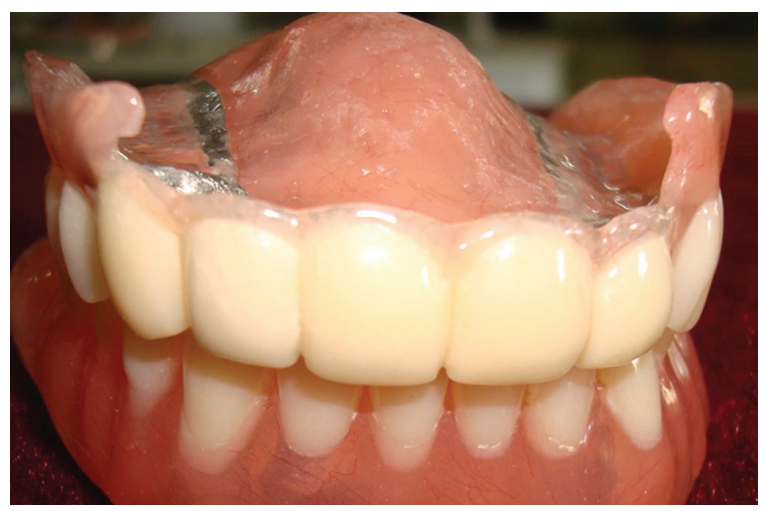

Fig. 5: Final prosthesis

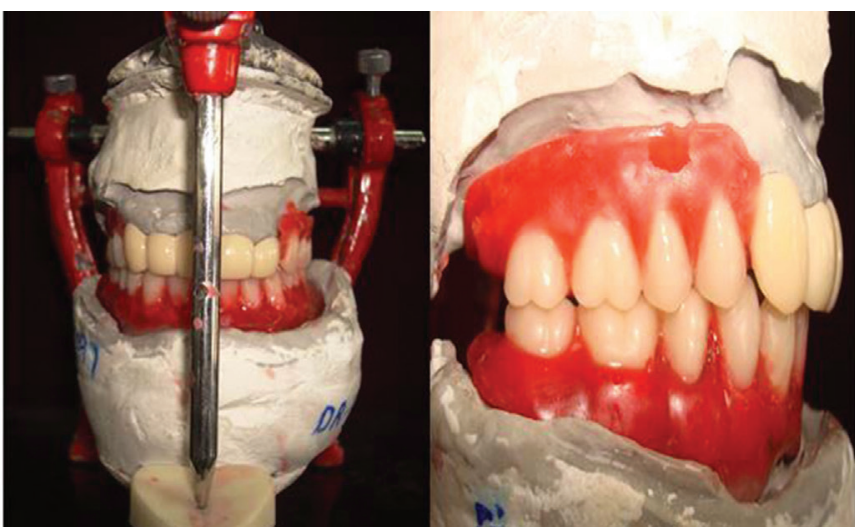

Fig. 4: Trial setting

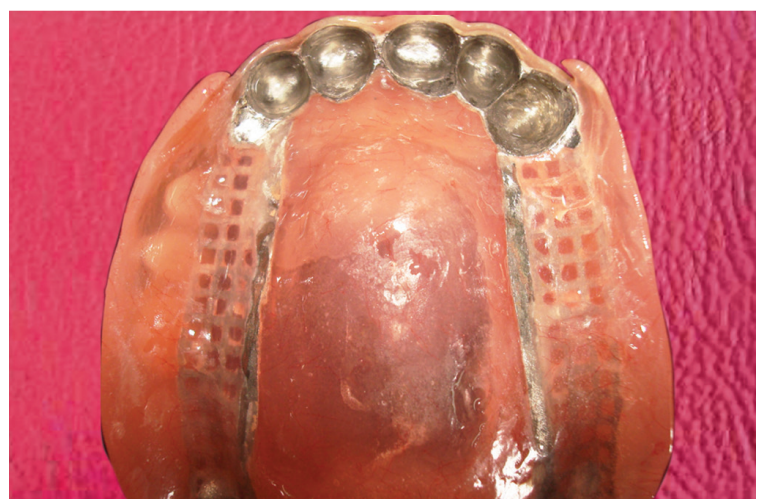

Fig. 6: Finished maxillary double-crown overdenture
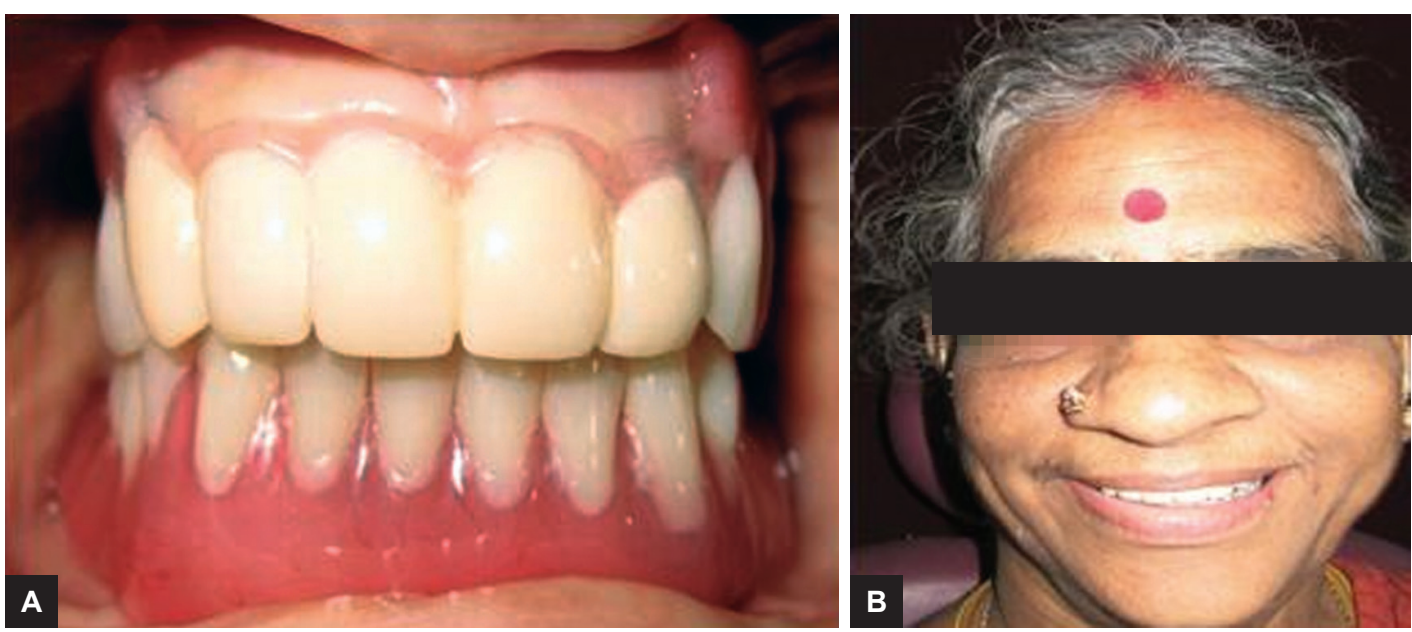

Figs 7A and B: Postoperative view: (A) Intraoral, and (B) extraoral

the cast framework of the maxillary arch and the acrylic denture base of the mandibular arch. Bite registration was done and the models were mounted on a semi-adjustable articulator. Acrylic teeth were set with the same shade of ceramic veneered over the secondary coping (Fig. 4). After verification of esthetics, function, and phonetics, the dentures were processed (Fig. 5). Flasking and acrylization of the maxillary cast with framework (Fig. 6) was done with special care by covering the ceramic surface with silicone putty in order to avoid any surface impurities due to investing plaster. Finishing and polishing of both upper and lower denture was done (Figs 7A and B). The completed prostheses were evaluated for function, esthetics, and phonetics.

\section{DISCUSSION}

The treatment modality for this patient was based on the functional and esthetic demands in conjunction with maintaining the longevity of the prosthesis. Periodontal status of the tooth involved in overdenture, should be evaluated if present in esthetic zone. Apart from this maxillary, alveolar ridge anatomy should be evaluated, 
such as undercuts because if present it will be difficult to obtain a single path of insertion along with the overlay coping. Excessive anterior block-out, in turn, results in bulky anterior flange, which will compromise the esthetics and speech. Moreover, the number of abutments in the coping that serve as a support for the overdenture should also be thought of, because the literature supports that a minimum of two abutment teeth should be splinted when the attachment prosthesis are used to make the stress pattern more favorable. ${ }^{8}$ Uniformly distributing the intimacy of contacts between the telescopic units and changing the taper angle and the height of coping provide better retentive control to telescopic retainers compared to conventional DEBs. ${ }^{9}$

With regard to the present case scenario, the periodontal involvement of the remaining dentition was minimal as evaluated by a specialist, clinically and radiographically giving a good long-term prognosis. After the initial phase of periodontal management with supragingival scaling along with oral hygiene instructions, the patient was well motivated. Emphasis on oral hygiene level can be one of the important factors as the status of such prosthesis and its usefulness to the patient solely depend on the continued retention of the underlying abutments. Moreover, in telescopic dentures the crowns were given to the abutment tooth, which may reduce the titling forces and promote axial loading, which stimulates periodontium. Conventional telescopic crowns achieve retention using friction of parallel-milled surfaces, and conical crowns exhibit retention using a wedging effect when completely seated, whereas the double crown with clearance fit exhibits minimal friction and no wedging during insertion and removal, thereby giving good success rates. ${ }^{10}$ The importance of maintaining recall visits, review, and patient motivation is mandatory for long-term success of the dentures. Basically such prosthesis will provide an indirect splinting influence and better oral hygiene maintenance.

\section{Specific Advantages of a Double-crown Overdenture}

- Creating a more natural emergence profile via ridge grip

- Psychological satisfaction of the patient
- Ample modification of the tooth anatomy in the esthetic zone based on the facial form

- Minimal frictional and wedging effect

- Rigid splinting action.

\section{Limitations}

- Periodic maintenance recall

- Extensive laboratory procedure

- More dental visits

- Difficulty on modification of retentive forces.

\section{CONCLUSION}

Double crowns if fabricated indigenously for individual cases would make attachments for overdenture effective; apart from the splinting of the abutment, it permits easy home care for oral hygiene maintenance, serving as an excellent treatment option for patients with reduced dentition.

\section{REFERENCES}

1. Kalpana C, Vamsi Prasad K. Seeing the unseen: preventive prosthodontics: use of overlay removable dental prosthesis. Ann Essences Dent 2010 Jul-Sep;2(3):44-49.

2. Prince IB. Conservation of the supporting mechanism. J Prosthet Dent 1965 Mar-Apr;15:327-338.

3. Yalisove IL. Crown and sleeve coping retainers for removable partial prosthesis. J Prosthet Dent 1966 Nov-Dec;16(6): 1069-1085.

4. Perel ML. Telescope dentures. J Prosthet Dent 1973 Feb;29(2): 151-156.

5. Langer A. Telescopic retainers for removable partial dentures. J Prosthet Dent 1981 Jan;45(1):37-43

6. Wenz HJ, Hertrampf K, Lehmann KM. Clinical longevity of removable partial dentures retained by telescopic crowns: outcome of the doublecrownwithclearancefit.IntJProsthodont 2001 May-Jun;14(3):207-213

7. Miller PD Jr. A classification of marginal tissue recession. Int J Periodontics Restorative Dent 1985;5(2):8-13.

8. Saito M, Miura Y, Notani K, Kawasaki T. Stress distribution of abutments and base displacement with precision attachment and telescopic crown-retained removable partial dentures. J Oral Rehabil 2003 May;30(5):482-487.

9. Ogata K, Ishii A, Shimizu K, Watanabe N. Longitudinal study on occlusal force distribution in lower distal-extension removable partial dentures with conus crown telescopic system. J Oral Rehabil 1993 Jul;20(4):385-339.

10. Besimo C, Graver G. A new concept of over denture with telescope crowns on osseointegrated implants. Int J Periodont Restorative Dent 1994 Dec;14(6):487. 